\title{
The role of hereditary nonpolyposis colorectal cancer in the management of familial ovarian cancer
}

Carolyn Farrell, MS, $\mathrm{NP}-\mathrm{C}^{1}$, Mollie Lyman, $M S^{1}$, Katherine Freitag, $B S^{1}$, Cathy Fahey, $B S^{2}$, M. Steven Piver, $M D^{2}$, and Kerry J. Rodabaugh, $M D^{2,3}$

\begin{abstract}
Purpose: Familial ovarian cancer is most often associated with hereditary breast and ovarian cancer, implicating mutations in the BRCA1 and BRCA2 genes. Hereditary nonpolyposis colorectal cancer, another common syndrome, is also associated with ovarian cancer and is caused by DNA mismatch repair genes. We sought to identify the role of hereditary nonpolyposis colorectal cancer in women with family histories of ovarian cancer. Methods: The likelihood of a genetic syndrome in 226 oophorectomized women in the Gilda Radner Familial Ovarian Cancer Registry was determined by pedigree analysis using clinical criteria and by calculating the probability of a mutation in genes responsible for hereditary breast and ovarian cancer and hereditary nonpolyposis colorectal cancer using available risk models. Results: Some $86 \%$ had a BRCA gene mutation likelihood of $7.8 \%$ or higher, warranting consideration of hereditary breast and ovarian cancer. Of the 32 women below this threshold, 4 (12.5\%) had family histories that met criteria for clinical diagnosis of hereditary nonpolyposis colorectal cancer. In addition, 16 women (7\%) with a BRCA mutation likelihood greater than $7.8 \%$ met clinical criteria for hereditary nonpolyposis colorectal cancer or warranted its inclusion in the differential diagnosis. Among all study respondents, $9 \%$ had family histories warranting consideration of hereditary nonpolyposis colorectal cancer. Conclusion: Hereditary nonpolyposis colorectal cancer should be considered in the differential diagnosis of women with family histories of ovarian cancer.
\end{abstract}

Genet Med 2006:8(10):653-657.

Inherited gene mutations account for $5 \%$ to $10 \%$ of all epithelial ovarian cancers. A positive family history is one of the most important risk factors for developing ovarian cancer. The genes most commonly associated with susceptibility to ovarian cancer are BRCA1 and BRCA2. Mutations in either gene confer a lifetime risk for ovarian cancer of $16 \%$ to $44 \%^{1,2}$ and a lifetime risk for breast cancer of $60 \%$ to $85 \% .{ }^{3}$ Because BRCA1 and BRCA2 mutations only account for up to $85 \%$ of families with inherited breast and/or ovarian cancer, other gene mutations are likely to be responsible for the remaining hereditary cases.

Hereditary nonpolyposis colorectal cancer (HNPCC) is also associated with an increased risk for ovarian cancer. Germline mutations in HNPCC-related genes (MLH1, MSH2, MSH6, and PMS2) confer risks for colorectal, endometrial, and ovarian cancer of up to $90 \%, 60 \%$, and $13 \%$, respectively. ${ }^{4}$ The level

\footnotetext{
From the ${ }^{1}$ Division of Clinical Genetics Service; ${ }^{2}$ Gilda Radner Familial Ovarian Cancer Registry; and ${ }^{3}$ Division of Gynecologic Oncology, Roswell Park Cancer Institute, Buffalo, New York.

Kerry J. Rodabaugh, MD, Roswell Park Cancer Institute, Elm and Carlton Streets, Buffalo, NY 14263.

Submitted for publication June 2, 2006.

Accepted for publication July 14, 2006.

There are no conflicts of interest in this article with any authors.

DOI: 10.1097/01.gim.0000241895.20390.c5
}

of ovarian cancer risk associated with HNPCC has not been widely appreciated, as is evidenced by the fact that the clinical criteria (Amsterdam; modified Amsterdam) for the diagnosis of HNPCC does not include ovarian cancer but does include stomach cancer, which carries essentially the same level of risk as ovarian cancer. ${ }^{5}$ In addition, the clinical diagnosis of HNPCC was confined to families with three or more affected individuals. ${ }^{6}$ On the basis of both family and molecular genetic studies, it became apparent that these criteria were too restrictive and resulted in missing persons/families at risk. ${ }^{7}$ Clinical criteria were therefore modified to include other cancers in the HNPCC spectrum, and although ovarian cancer is not among these criteria, there is recognition of strong and consistent evidence that ovarian cancer is in the HNPCC tumor spectrum. ${ }^{8}$

We hypothesized that although women with family histories of ovarian cancer are at increased risk for the development of ovarian cancer on the basis of family history alone, and many have an increased likelihood of carrying a germline mutation in either the BRCA1 or BRCA2 genes, there may be a significant number whose cancer risk is the result of other hereditary ovarian cancer-associated syndromes, specifically HNPCC. We further hypothesized that because syndromes related to mutations in BRCA1 and BRCA2 are more common, they are often the only syndromes considered in risk analysis. Thus, some women and their health care providers may not have all the information necessary regarding their individual risk for 
ovarian (or other) cancer(s) to make informed decisions regarding genetic testing and risk-management approaches. In an effort to address these issues, we surveyed unaffected members of the Gilda Radner Familial Ovarian Cancer Registry (GRFOCR) who had previously undergone oophorectomy.

\section{METHODS}

This study invited participation, by questionnaire, of women in the GRFOCR who had undergone previous oophorectomy between 1981 and 2002. Inclusion in the Registry requires a family history of ovarian cancer, and the majority of women had at least two close (first- or second-degree) relatives with ovarian cancer. A total of 317 questionnaires were sent out, with a response rate of 63\% (199). This questionnaire supplemented a questionnaire previously sent to these same oophorectomized women in conjunction with an earlier study investigating surgical procedures. Thus, although respondents to the current questionnaire totaled 199, some data from the previous study are relevant to the current study and included here, making $\mathrm{N}=226$ (71\%) for some variables. Survey questions addressed the following topics: types of cancers, age at diagnosis for the participant and relatives, reason for oophorectomy, and whether the participant had received genetic counseling or testing. Ancestry was assessed because individuals of Ashkenazi Jewish ancestry have a higher likelihood (2.5\%) of having one of three specific BRCA1 or BRCA2 gene mutations associated with hereditary breast and ovarian cancer (HBOC). ${ }^{9}$

All of the women had previously submitted a pedigree as part of their participation in the GRFOCR. These pedigrees were reviewed for personal and family history of malignancy by a genetic counselor to clinically classify cases into possible hereditary ovarian cancer syndromes, HBOC or HNPCC. Maternal and paternal sides were classified separately; siblings of the proband were considered both on the maternal and the paternal sides. Pedigrees with only breast cancer (age $<50$ years), or both breast and ovarian cancer in the same lineage, were considered in the context of HBOC. Alternatively, modified Amsterdam criteria-at least three relatives with HNPCC-related cancer (e.g., colon, endometrial, ovarian), one of whom is a first-degree relative of the other two; at least two successive generations affected; and at least one individual diagnosed before the age of 50 years ${ }^{10}$ - were used for the clinical diagnosis of HNPCC. Furthermore, those lacking only one criteria for HNPCC were noted because experience has shown that in some of these families, HNPCC can be confirmed on a molecular basis, which led to the modification (broader, less stringent) of the guidelines for suspecting and testing for HNPCC. ${ }^{7}$ Families with only ovarian cancer or ovarian cancer plus other cancers who did not fit into these classifications were considered in the context of both HBOC and HNPCC and were grouped separately.

The clinical classification approach was used because this allowed for consideration of aspects of medical history not integrated into mutation likelihood models (e.g., third-degree relatives or families with HNPCC-associated cancers yet no colon cancer), thus accounting for differences between the clinical assessment and the statistical likelihood of identifying a gene mutation. The clinical classification provided the foundation for application of the statistical mutation likelihood programs to appropriate cases.

Risk for BRCA1 or BRCA2 mutations was assessed using Myriad Tables and BRCAPRO. Myriad Tables were developed from data by Myriad Genetics Laboratories (Salt Lake City, UT) with the purpose to provide the likelihood of a BRCA1 or BRCA2 mutation based on results from testing more than 10,000 individuals and applying logistic regression based on various characteristics of family history. These tables include frequencies and percentages specific to persons of Ashkenazi Jewish ancestry and all other persons. They include consideration of first- and second-degree relatives with an ovarian cancer diagnosis at any age, breast cancer at less than 50 years of age, or both; and for the proband, diagnosis of ovarian cancer and/or breast cancer at any age. ${ }^{11}$

The BRCAPRO model is part of the CancerGene software tool (University of Texas Southwestern Medical Center at Dallas, Dallas, TX) developed for evaluating mutation likelihoods in families suggestive of hereditary cancer syndromes. BRCAPRO invokes a statistical method for calculating a person's probability of carrying a deleterious mutation in either BRCA1 or BRCA2. Calculations are made on the basis of the individual's cancer status and that person's family history of breast and ovarian cancer. It uses the autosomal dominant Mendelian inheritance of the BRCA1 and BRCA2 genes, and incorporates prevalence and penetrance using results published by Iversen. ${ }^{12}$ It also integrates information regarding gender, current age or age at death, exact relationship to the proband, diagnoses of breast and/or ovarian cancer, and age at diagnosis for the proband and each first- and second-degree relative. ${ }^{13}$ Ashkenazi Jewish ancestry is also taken into account. ${ }^{14}$ CancerGene can also calculate the likelihood of carrying a mutation in one of the two most common HNPCC-related genes (MLH1 and $\mathrm{MSH} 2$ ) using a similar approach. A limitation in this model exists in that it only allows for computation of HNPCC mutation likelihood if there is a colon cancer in the family. ${ }^{15}$

If only part of the patient's family history was known, a conservative approach for these calculations was taken. For example, if the age at diagnosis of breast cancer in the family was unknown, the age supplied to BRCAPRO was greater than 50 years. The median sensitivity of BRCAPRO for identifying mutation carriers has been found to be $94 \% .^{16}$

Data collected from the surveys were entered into a database and then analyzed.

\section{RESULTS}

Demographic data and response frequencies for participants, all of whom had a personal and/or family history of ovarian cancer, and relevant variables are listed in Table 1. Clinical cancer syndrome classifications (HBOC and HNPCC), based on personal and family cancer history as described in the "Methods" 
Table 1

Demographics and response frequencies

\section{Population characteristics}

\section{White}

Jewish

Personal history of cancer

Prophylactic oophorectomy

Personal perception of risk

High risk

Medium risk

Low risk

Genetic counseling/testing

$73 \%(165 / 226)$ did not have genetic counseling

$80 \%(181 / 226)$ did not have genetic testing

section, were assessed. The results of classification into syndromes consistent with HBOC and HNPCC, evaluated separately from the maternal and paternal perspectives, demonstrate similar percentages (Fig. 1).

According to BRCAPRO and Myriad laboratory data tables, $86 \%(194 / 226)$ had a BRCA1 or BRCA2 mutation likelihood of $7.8 \%$ or greater as calculated by at least one of these models (compared with a general population risk of $<1 \%$ ); $7.8 \%$ was used as the lower threshold suggestive of a breast/ovarian cancer syndrome because this correlates with the mutation likelihood probability for a woman with a diagnosis of early-onset breast cancer (age $<50$ years), an accepted "standard" for high risk at the time of this study, ${ }^{17}$ as well as a significantly greater risk than for the gen-

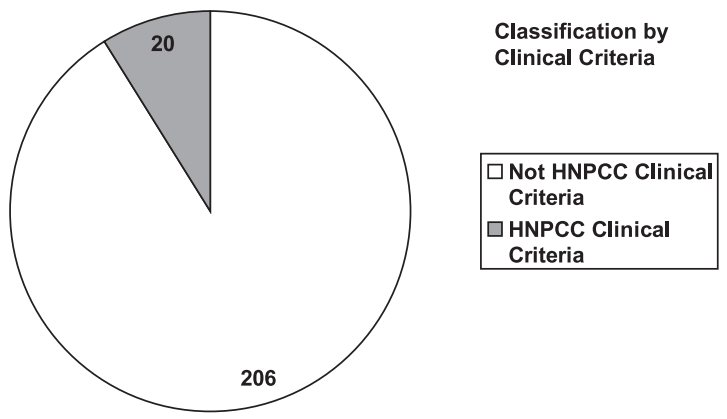

Fig. 2. Of the 226 patients surveyed, 20 met (or lacked only one) clinical criteria for HNPCC. HNPCC, hereditary nonpolyposis colorectal cancer.

eral population. ${ }^{18}$ The mutation likelihood results for BRCAPRO ranged from $0.1 \%$ to $100 \%$ with a mean of $21 \%$, whereas Myriad ranged from $3 \%$ to $90 \%$ with a mean of $16 \%$.

Independent of BRCA mutation likelihood, 20 of the 226 respondents (9\%) met, or lacked only one, criteria for the clinical diagnosis of HNPCC using modified Amsterdam criteria (Fig. 2). An HNPCC mutation likelihood could be computed for only 16 of these 20 respondents because CancerGene requires the presence of colon cancer in the family to make a calculation. The average HNPCC mutation likelihood was $26 \%$ with values ranging from $1 \%$ to $91 \%$. From a practical perspective, of these 20 women identified clinically to be at risk for HNPCC, only 3 (14\%) indicated concern regarding their risk of developing endometrial cancer and $16(76 \%)$ had not seen a genetic counselor.

Of the 32 women (14\%) with a low BRCA mutation likelihood $(<7.8 \%$ in all models), an HNPCC mutation likelihood could be computed for 9 , with the average mutation likelihood of $8 \%$,

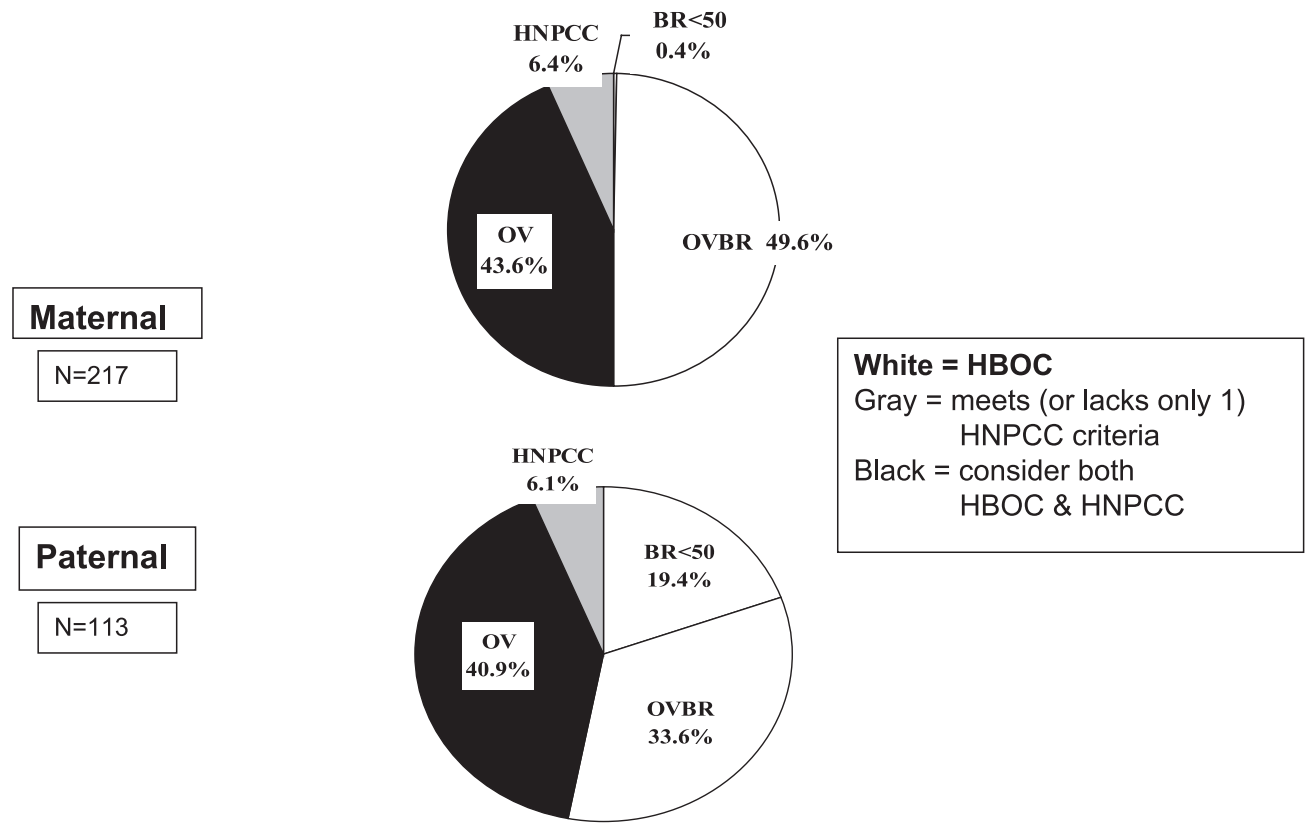

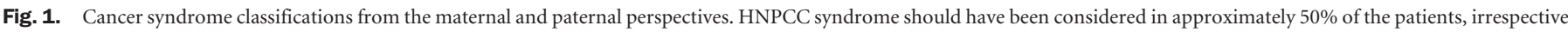
of the lineage evaluated. HNPCC, hereditary nonpolyposis colorectal cancer; HBOC, hereditary breast and ovarian cancer. 

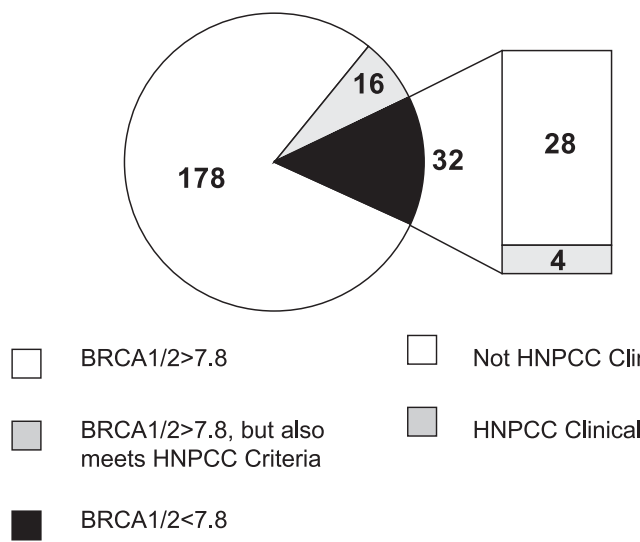

\begin{abstract}
BRCA $1 / 2>7.8$
BRCA1/2>7.8, but also
\end{abstract} meets HNPCC Criteria

BRCA $1 / 2<7.8$

Fig. 3. A total of 194 patients had a BRCA mutation likelihood of greater than $7.8 \%$, and 16 of these patients met (or lacked only one) HNPCC clinical criteria. Of the 32 patients with a BRCA mutation likelihood less than 7.8\%, 4 met clinical criteria for HNPCC. HNPCC, hereditary nonpolyposis colorectal cancer.

ranging from $1 \%$ to $23 \%$. On the basis of clinical assessment, 4 of $32(12.5 \%)$ were diagnostic of HNPCC (Fig. 3), yet not all could have a mutation likelihood generated by CancerGene because of a lack of colon cancer in the family.

\section{DISCUSSION}

Persons considered at increased risk for ovarian cancer use oophorectomy as an approach to risk management. Accurately recognizing inherited cancer risk, the corresponding hereditary syndrome, and the genetic cause of the particular syndrome (e.g., BRCA1/BRCA2 vs. HNPCC-associated genes) is critical to appropriate cancer screening recommendations and recommendation for surgical intervention (e.g., oophorectomy vs. oophorectomy and hysterectomy). When investigating self-perceived risk, Bluman et al. ${ }^{19}$ found that most women overestimate their chances of having a mutation in BRCA1 or BRCA2 when compared with estimates calculated from a risk model. The perception of increased risk for ovarian cancer, based on having an affected relative, was the most frequent response in the decision for oophorectomy for the women in this study. Overestimation of cancer risk and a lack of accurate knowledge may affect a woman's risk-management and treatment decisions, including the decision to undergo a prophylactic oophorectomy. A method to accurately determine risk for ovarian cancer is therefore critical to both individuals at risk and their health care providers for medical and surgical risk-management decisions.

Current approaches to identify increased risk for the development of ovarian cancer include assessment of family history and determination of likelihood of gene mutations using riskassessment models. In the present study, participants from the GRFOCR were at increased risk because most had at least two relatives with ovarian cancer. All participants in this study had undergone oophorectomy. It is noteworthy, however, that if ovarian cancer risk were the result of a heritable genetic mutation, then statistically, 50\% of women in these families would not have inherited the mutation, would not be at increased risk for ovarian cancer, and thus would not have been candidates for prophylactic surgery. This endorses the importance of genetic counseling, risk assessment and consideration of genetic testing of an appropriate affected relative, and/or direct testing of the unaffected at risk (in specific populations) before proceeding with oophorectomy.

Use of risk-assessment models add an objective, consistent approach to determination of risk. In this study, $86 \%$ of women were identified at statistically increased risk for a BRCA1 or BRCA 2 mutation (likelihood of $7.8 \%$ or higher, substantially greater than that of the general population) in at least one model. Although these models and computed risk figures are based on published studies and large data sets, they may underestimate or inaccurately assign risk for several reasons. Their limitations are based on limited experience, and with the exception of the BRCAPRO model, they only consider HBOC syndrome. Fourteen percent of this study population had a calculated BRCA1 or BRCA2 mutation likelihood less than $7.8 \%$, yet they had a family history of ovarian cancer and are still considered at increased risk for medical management purposes. If the likelihood of identifying a BRCA1 or BRCA2 gene mutation is the only variable used for offering genetic evaluation or testing, or confirming increased risk for ovarian cancer, these women may be managed inappropriately. It is possible that these persons are at increased ovarian cancer risk because of a cancer-associated genetic syndrome other than HBOC, for example, HNPCC, making them potential candidates for further genetic evaluation, counseling, and/or testing.

Members of HNPCC families have a $12 \%$ to $13 \%$ risk for developing ovarian cancer; ${ }^{4}$ thus, consideration of HNPCC in the differential diagnosis of familial ovarian cancer is warranted. In a study of 116 unselected patients with ovarian cancer, Rubin et al. ${ }^{20}$ identified two germline HNPCC mutations. Both of these patients had personal histories of a separate HNPCC-associated cancer. Lu et al. ${ }^{21}$ studied 117 women with dual primary cancers from 223 families who fulfilled Amsterdam criteria for HNPCC. In 10\% of these cases, ovarian cancer was a sentinel cancer for the patient (either diagnosed first or synchronously).

In our study, we found that of those whose likelihood of a BRCA1 or BRCA2 mutation was less than $7.8 \%$, several had family histories that met criteria or were suggestive of the clinical diagnosis of HNPCC. When an HNPCC mutation likelihood could be computed, an average mutation likelihood of $8 \%$ was found. This is increased from that of the general population $(<1 \%)$ and supports consideration of HNPCC. This level of risk is in the same order of risk/likelihood as that considered significant for testing for a BRCA1 or BRCA2 mutation.

It is important to note that in the current study, using the clinical approach to pedigree analysis, there were 20 women (9\%) who had family histories that were diagnostic or suggestive of HNPCC, yet 16 of these women also had a BRCA1 or BRCA2 mutation likelihood of $7.8 \%$ or greater. Thus, if genetic testing were offered to these women, it would likely be directed 
to the BRCA1 and BRCA2 genes, although HNPCC testing may be the more appropriate test. Because of the absence of colon cancer in the family, CancerGene could not be computed for all, but in those able to be computed, an average HNPCC mutation likelihood of $26 \%$ was found. It is a limitation of CancerGene that an HNPCC mutation likelihood in families lacking colon cancer but with multiple other HNPCC-related malignancies (e.g., endometrial, ovarian) cannot be calculated using this model. In addition, this model only considers up to second-degree relatives and only calculates mutation likelihood based on the two most common HNPCC genes (MLH1 and MSH2). Consequently, CancerGene may underestimate HNPCC mutation likelihood, indirectly suggesting exclusion of HNPCC in the differential diagnosis.

Consideration of HNPCC is important in risk-management decisions in persons/families with ovarian cancer because there is up to a $60 \%$ risk of endometrial cancer and a $54 \%$ risk of colon cancer in affected women. ${ }^{5}$ This is relevant to decisions regarding gynecologic management, including surveillance and consideration of hysterectomy in addition to oophorectomy, as well as to recommendations of colonoscopy for colon cancer screening. Of the women in this study who met or lacked only one criteria for HNPCC, only three (15\%) indicated concern regarding their risk for endometrial cancer, and $16(76 \%)$ had not seen a genetic counselor. In this study, $9 \%$ of participants had a family history of ovarian cancer that met clinical criteria (or lacked only one criteria) warranting consideration of HNPCC. These data support inclusion of HNPCC in the differential diagnosis of inherited risk for ovarian cancer and consideration of genetic consultation concerning their cancer risk, genetic testing, and discussion of management options.

Women presenting with a family history of ovarian cancer, whether maternal or paternal, are not only considered at increased statistical ovarian cancer risk, but warrant consideration of cancer-associated genetic syndromes such as HBOC and HNPCC. In the current study, $42 \%$ of the respondents had a family history of only ovarian cancer, or ovarian and another cancer that did not "fit" into the clinical classifications based on features of known cancer genetic syndromes. Risk-assessment models are useful, but as discussed, have significant limitations. Genetic assessment, counseling, and possible testing are important elements of cancer risk determination, whether provided by the primary care practitioner or in conjunction with a genetic counselor or specialist. Genetic testing must be directed to appropriate genes to ensure accurate interpretation of results and potentially confirm or rule out increased risk.
This includes the consideration of HNPCC in women who have family histories of ovarian cancer, because ultimately the assessment impacts confirmation or exclusion of inherited risk, medical management, cancer screening recommendations, and surgical decision-making, including the need for prophylactic oophorectomy and/or hysterectomy.

\section{References}

1. Easton DF, Bishop DT, Ford D, Crockford GP. Genetic linkage analysis in familial breast and ovarian cancer: results from 214 families. The Breast Cancer Linkage Consortium. Am J Hum Genet 1993;52:678-701.

2. Ford D, Easton DF, Bishop DT, Narod SA, et al. Risks of cancer in BRCA1-mutation carriers. Breast Cancer Linkage Consortium. Lancet 1994;343:692-695.

3. Wooster R, Weber BL. Breast and ovarian cancer. NEngl J Med 2003;348:2339-2347.

4. Lynch HT, Casey MJ, Shaw TG, Lynch JF. Hereditary factors in gynecologic cancer. Oncologist 1998;3:319-338.

5. Aarnio M, Sankila R, Pukkala E, Salovaara R, et al. Cancer risk in mutation carriers of DNA-mismatch-repair genes. Int J Cancer 1999;81:214-218.

6. Vasen HF. Clinical diagnosis and management of hereditary colorectal cancer syndromes. J Clin Oncol 2000;18:81S-92S.

7. Umar A, Boland CR, Terdiman JP, Syngal S, et al. Revised Bethesda Guidelines for hereditary nonpolyposis colorectal cancer (Lynch syndrome) and microsatellite instability. J Natl Cancer Inst 2004;96:261-268.

8. Watson P, Riley B. The tumor spectrum in the Lynch syndrome. Fam Cancer 2005; 4:245-248.

9. Struewing JP, Hartge P, Wacholder S, Baker SM, et al. The risk of cancer associated with specific mutations of BRCA1 and BRCA2 among Ashkenazi Jews. N Engl J Med 1997;336:1401-1408.

10. Rodriguez-Bigas MA, Boland CR, Hamilton SR, Henson DE, et al. A National Cancer Institute Workshop on Hereditary Nonpolyposis Colorectal Cancer Syndrome: meeting highlights and Bethesda guidelines. J Natl Cancer Inst 1997;89:1758-1762.

11. Frank TS, Deffenbaugh AM, Reid JE, Hulick M, et al. Clinical characteristics of individuals with germline mutations in BRCA1 and BRCA2: analysis of 10,000 individuals. J Clin Oncol 2002;20:1480-1490.

12. Iversen ES. Genetic susceptibility and survival: application to breast cancer. J Am Stat Assoc 2000;95:28-42.

13. Berry DA, Parmigiani G, Sanchez J, Schildkraut J, et al. Probability of carrying a mutation of breast-ovarian cancer gene BRCAl based on family history. J Nat Cancer Inst 1997;89:227-238.

14. Parmigiani G, Berry D, Aguilar O. Determining carrier probabilities for breast cancer-susceptibility genes BRCA1 and BRCA2. Am J Hum Genet 1998;62:145-158.

15. Wijnen JT, Vasen HF, Khan PM, Zwinderman AH, et al. Clinical findings with implications for genetic testing in families with clustering of colorectal cancer. $N$ Engl J Med 1998;339:511-518.

16. Euhus DM, Smith KC, Robinson L, Stucky A, et al. Pretest prediction of BRCAl or BRCA2 mutation by risk counselors and the computer model BRCAPRO. J Natl Cancer Inst 2002;94:844-851.

17. Kutner SE. Breast cancer genetics and managed care. The Kaiser Permanente experience. Cancer 1999;86:2570-2574.

18. Rahman N, Stratton MR. The genetics of breast cancer susceptibility. Annu Rev Genet 1998;32:95-121.

19. Bluman LG, Rimer BK, Berry DA, Borstelmann N, et al. Attitudes, knowledge, and risk perceptions of women with breast and/or ovarian cancer considering testing for BRCA1 and BRCA2. J Clin Oncol 1999;17:1040-1046.

20. Rubin SC, Blackwood MA, Bandera C, Behbakht K, et al. BRCA1, BRCA2, and hereditary nonpolyposis colorectal cancer gene mutations in an unselected ovarian cancer population: relationship to family history and implications for genetic testing. Am J Obstet Gynecol 1998;178:670-677.

21. Lu KH, Dinh M, Kohlmann W, Watson P, et al. Gynecologic cancer as a "sentinel cancer" for women with hereditary nonpolyposis colorectal cancer syndrome. $\mathrm{Ob}$ stet Gynecol 2005;105:569-574. 